\title{
Reducing Magnetic Noise of an Unmanned Aerial Vehicle for High-Quality Magnetic Surveys
}

\author{
Boris Sterligov and Sergei Cherkasov \\ Vernadsky State Geological Museum, Russian Academy of Sciences, 11/11 Mokhovaya Street, Moscow 125009, Russia \\ Correspondence should be addressed to Boris Sterligov; b.sterligov@sgm.ru
}

Received 23 November 2015; Revised 15 February 2016; Accepted 15 February 2016

Academic Editor: Jean-Pierre Burg

Copyright (C) 2016 B. Sterligov and S. Cherkasov. This is an open access article distributed under the Creative Commons Attribution License, which permits unrestricted use, distribution, and reproduction in any medium, provided the original work is properly cited.

The use of light and ultralight unmanned aerial vehicles (UAVs) for magnetic data acquisition can be efficient for resolving multiple geological and engineering tasks including geological mapping, ore deposits' prospecting, and pipelines' monitoring. The accuracy of the aeromagnetic data acquired using UAV depends mainly on deviation noise of electric devices (engine, servos, etc.). The goal of this research is to develop a nonmagnetic unmanned aerial platform (NUAP) for high-quality magnetic surveys. Considering parameters of regional and local magnetic survey, a fixed-wing UAV suits geological tasks better for plain area and copter type for hills and mountains. Analysis of the experimental magnetic anomalies produced by a serial light fixed-wing UAV and subsequent magnetic and aerodynamic modeling demonstrates a capacity of NUAP with internal combustion engine carrying an atomic magnetic sensor mounted on the UAV wings to facilitate a high-quality magnetic survey.

\section{Introduction}

Through the last decade, various approaches to the use of $\mathrm{UAV}$ as a platform for magnetic surveys have been tried as with copter [1] and with fixed-wing [2] UAVs. Applicability of different platforms is reasoned by the goals of the survey, characteristics of the area and grid configuration, and amplitudes of the magnetic anomalies. The UAV magnetic survey stays in between a traditional aeromagnetic (using an aircraft or helicopter) and ground magnetic surveys in terms of productivity, accuracy, and the flight height above the surface. Consequently, when aeromagnetic survey is preferable for small-to-medium scale mapping and the ground one for high-precision large-scale survey, use of UAV can be competitive for geological mapping and ore deposits prospecting at 1:5000-1:50000 scales. Usually, areas for such surveys range from the first square kilometers up to 200 sq.km. The advantage of UAV in comparison with the ground surveys is, obviously, a speed of the survey. At the same time, light UAV (up to $10 \mathrm{~kg}$ ) does not need special infrastructure necessary for traditional aircraft. However, to compete with traditional aeromagnetic and ground systems, the UAV should be characterized by corresponding magnetic error of the survey, which, for a traditional aerial survey, does not exceed $2 \mathrm{nT}$.

To create the NUAP on the base of a light fixed-wing UAV weighting below $10 \mathrm{~kg}$, taking-off from catapult, and landing with a parachute, the serial commercially available UAV "Geoscan-201" (Russia) was chosen for initial experiments (Figure 1). The NUAP's magnetic noise should be characterized by gradient not exceeding $1 \mathrm{nT} / \mathrm{m}$ at the location of the sensor. The previous studies considered several possibilities to resolve this problem [2-5]. One of the most popular approaches is based on a passive compensation of magnetic noise, which comes from traditional aeromagnetic surveys. Realization of such an approach includes an additional special three-ax fluxgate magnetometer, installed close to magnetic elements of a platform, and postprocessing noise magnetic field compensation with special algorithms. Another possibility is to maximize distance from the sensor to the platform's magnetic elements (usually used on helicopters) using a flexible cable or telescopic rod. Unfortunately, both solutions are hardly applicable for a light UAV platform as the first one adds a sufficient weight and the second one makes UAV and the sensor movement unstable, which causes additional error to the positioning of the magnetic measurements. 


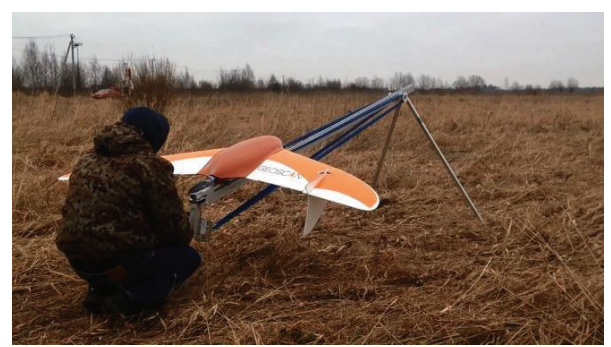

FIGURE 1: Launching UAV from catapult.

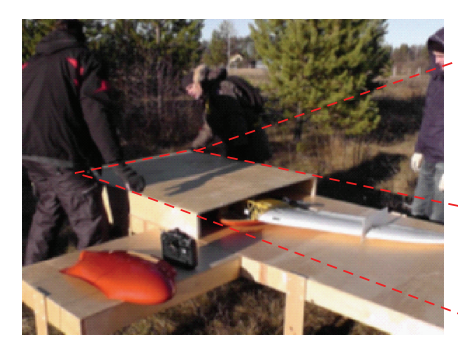

(a)

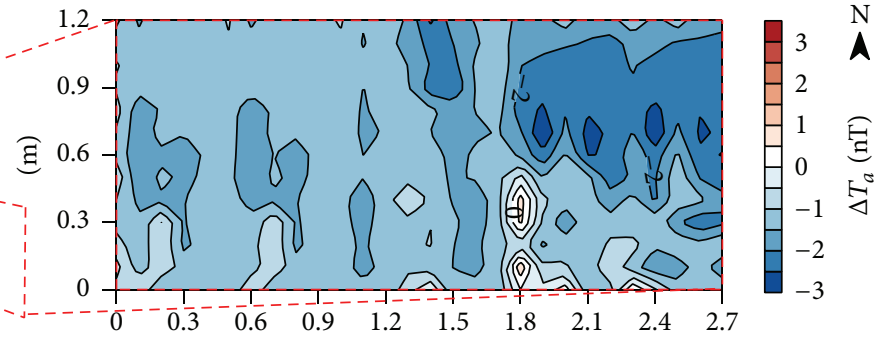

(m)

Contours step: $0.5 \mathrm{nT}$

(b)

FIgURE 2: Special stand for experiments: (a) mount UAV; (b) background magnetic field $\Delta T_{a}$ (top level without UAV).

The paper includes experimental data on the UAV's magnetic field, magnetic and aerodynamic mathematical modeling of NUAP, and description of the resulting concept and prototype.

\section{Experiment}

The goal of the experiment is to obtain the parameters of UAV magnetic sources in static and dynamic state for mathematical modeling needed to obtain an optimal design of the NUAP. The static and dynamic magnetic fields induced by UAV "Geoscan-201" components have been measured using special nonmagnetic stand $(120 \times 270 \mathrm{~cm})$ and atomic scalar magnetometers MMPOS-1 (Russia) and Geometrics G-858 (Canada) (Figure 2). To remove the background magnetic field, the measurements on the upper level of the stand have been initially executed without UAV by grid $10 \times$ $10 \mathrm{~cm}$ (Figure 2(b)). The amplitude of background magnetic anomalies of the stand is below $1 \mathrm{nT} / \mathrm{m}$.

2.1. Static Experiment. The objective of static experiment is to outline UAVs magnetic sources and their parameters. For the study, the UAV was fixed at the lower level of the stand, and the regular grid magnetic data of $10 \times 10 \mathrm{~cm}$ has been measured with a switched-off engine and electronics for four azimuths of the UAV longitudinal axis (north, west, south, and east). To obtain anomalous magnetic field, the geomagnetic variations and background magnetic field were considered. Analysis of the results clearly demonstrates the ends of UAV's wings as the most favorable sensor's locations (Figure 3). The magnetic deviation at nose cone of UAV (yellow points on Figure 3 ) is below $35 \mathrm{nT}$ and the horizontal gradient is $200 \mathrm{nT} / \mathrm{m}$ (Figure 4), which makes this location unsuitable for magnetic sensors. The magnetic deviation at two points on the end of UAV's wings (red and blue points on Figure 3 ) is below $2 \mathrm{nT}$ and the horizontal gradient does not extend above $10 \mathrm{nT} / \mathrm{m}$ (Figure 4).

2.2. Dynamic Experiment. The dynamic experiment targets variable magnetic fields induced by the UAV's electric devices: electroengine, servos, and electronics. The "Geoscan 201" uses electroengine Hyperion model Z4035-14 with titan 85 A high-voltage electronic speed controller weighting $284 \mathrm{~g}$. The servos are HS-65MG micrometal gear, with weight of $12 \mathrm{~g}$. Other electronics, such as GPS, antenna, and battery, provide very low magnetic noise. The experiment is very important in terms of the electroengine's influence evaluation. The rotor part of the engine contains magnetic masses rotating at frequency up to $30 \mathrm{KHz}$. Work frequency of the sensor is $50 \mathrm{~Hz}$ at maximum, and the filtering of magnetic noise generated by engine is impossible. The resulting magnetic fields of the different engine's regimes at the ends of the wings are shown in Figure 5. The average value of the magnetic noise produced by the functioning engine is rather stable and does not exceed $3 \mathrm{nT}$ (Figures 5(a), 5(b), 5(c), and 5(e)). The magnetic noise of servos is not more than $1 \mathrm{nT}$ for both sensor locations-left and right UAV wings (Figure 5(d)). The most interesting thing is field behavior at 


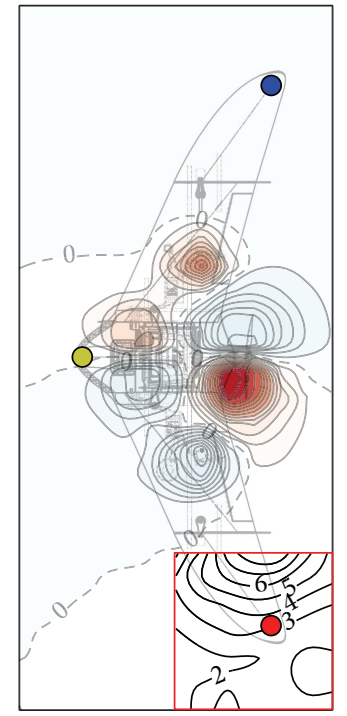

(a)

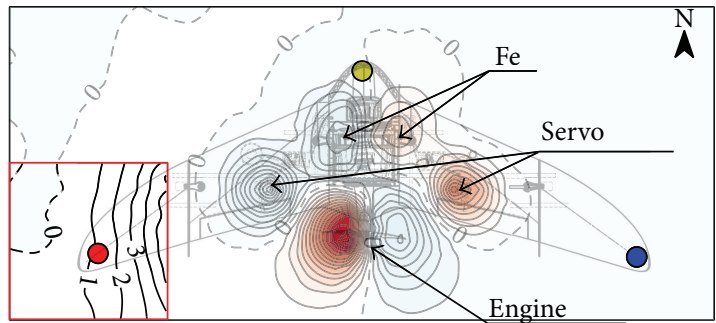

(b)

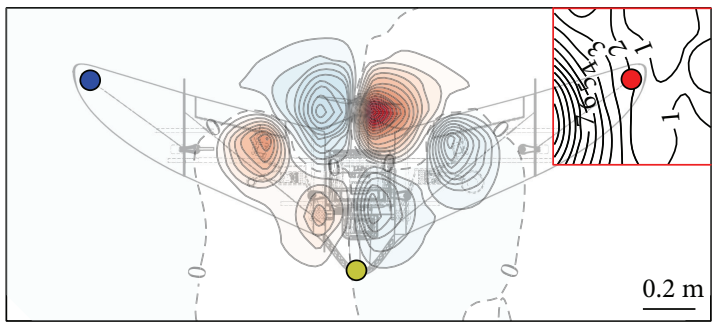

(c)
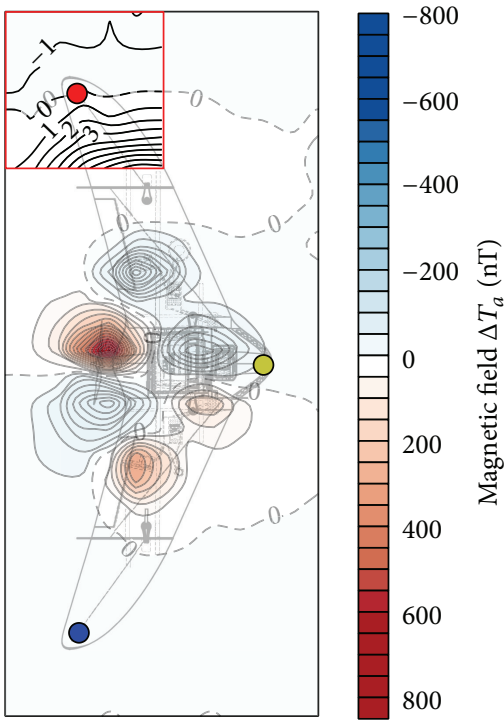

Contours step: $0.5 \mathrm{nT}$

(in red box: $1 \mathrm{nT}$ )

(d)

FIgURE 3: Magnetic field $\Delta T_{a}$ of UAV for its different orientation at the Earth's magnetic field: (a) west; (b) north; (c) south; (d) east.

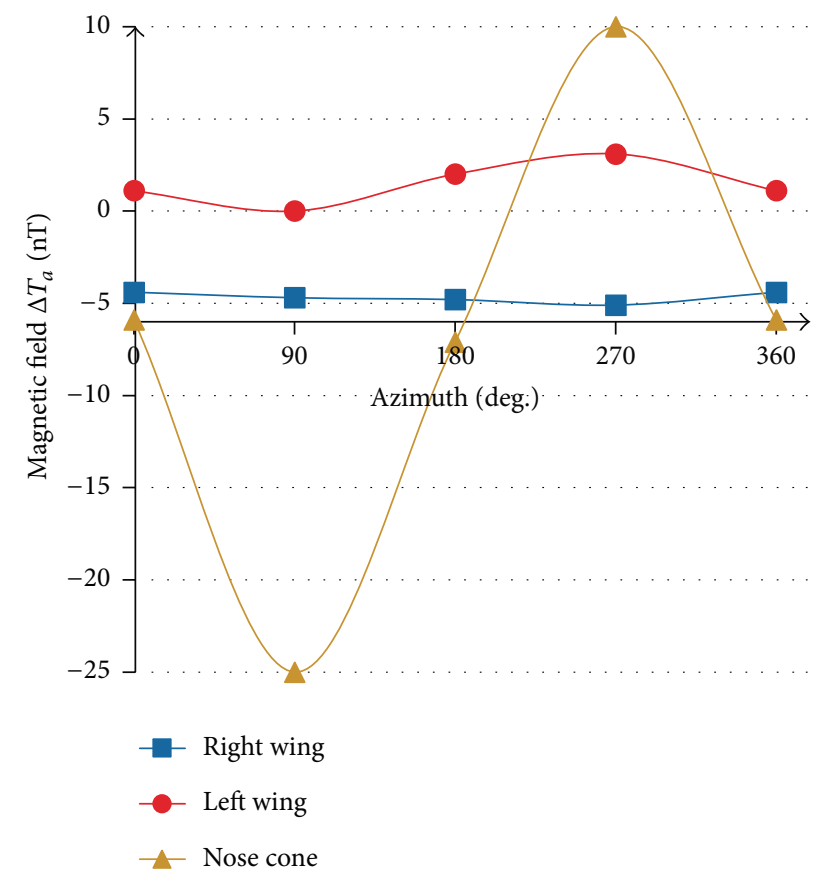

FigURE 4: Magnetic deviation of UAV (right wing: blue points on Figure 3; left wing: red points on Figure 3; nose cone: yellow points on Figure 3).

engine power off mode (Figure 5(f)). The abrupt change of the magnetic field corresponds to change position of magnets of electroengine before and after engine work (the graph in Figure 5(f) consists of data received between changes in modes of electroengine power). Thus, the electroengine can provide magnetic noise with amplitudes more than $10 \mathrm{nT}$.
The results of experiments have shown that the maximum noise magnetic field amplitude at the ends of UAV wings is $3 \mathrm{nT}$ for the various power of electroengine and unstable level of noise. Such a high value together with UAV magnetic deviation does not allow the use of an electroengine without passive field compensation. Servos as well represent the microelectroengine, but their operation does not produce a noticeable magnetic field at the distance over $1 \mathrm{~m}$.

\section{Magnetic and Aerodynamic Mathematical Modeling}

The analyses of static magnetic field of UAV demonstrate five sources of magnetic noise (Figure 6):

(1) Electroengine, producing a magnetic anomaly with amplitude up to $800 \mathrm{nT}$.

(2) Two servos (left and right), up to $600 \mathrm{nT}$.

(3) Ferromagnetic elements located at the frontal part of the UAV, up to $300 \mathrm{nT}$.

The total UAV's magnetic field can be represented by the sum of a number of dipole fields. Thus, for an electrical device producing magnetic field, the latest may be considered as being produced by a point source if the distance from the device is greater than 3 times the largest dimension of the device [6]. The magnetic field created by a dipole is given by vector described as [7]

$$
\bar{B}=\frac{\mu_{0}}{4 \pi}\left[-\frac{\bar{M}}{R^{3}}+\frac{3(\bar{M} \cdot \bar{R}) \bar{R}}{R^{5}}\right],
$$




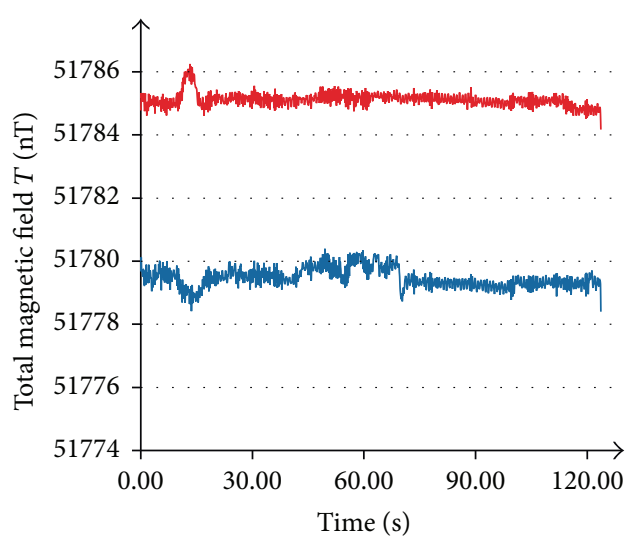

(a)

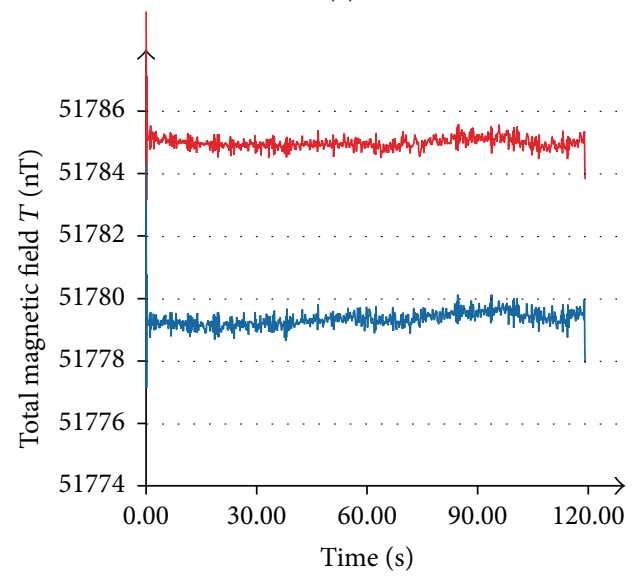

(c)

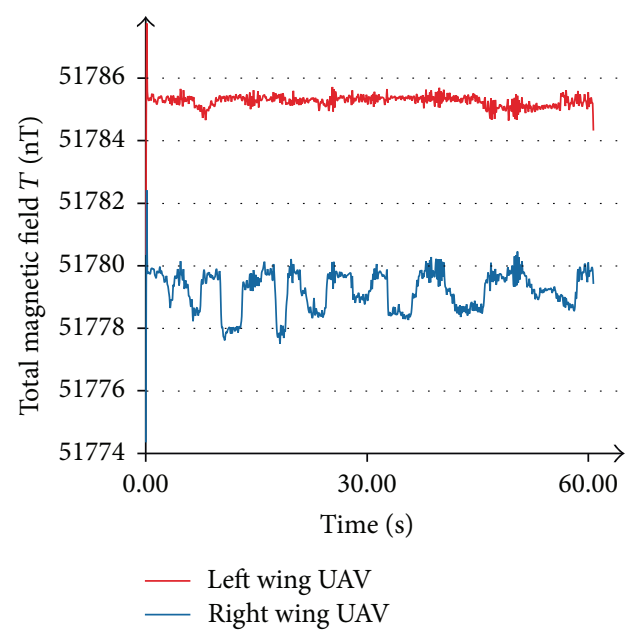

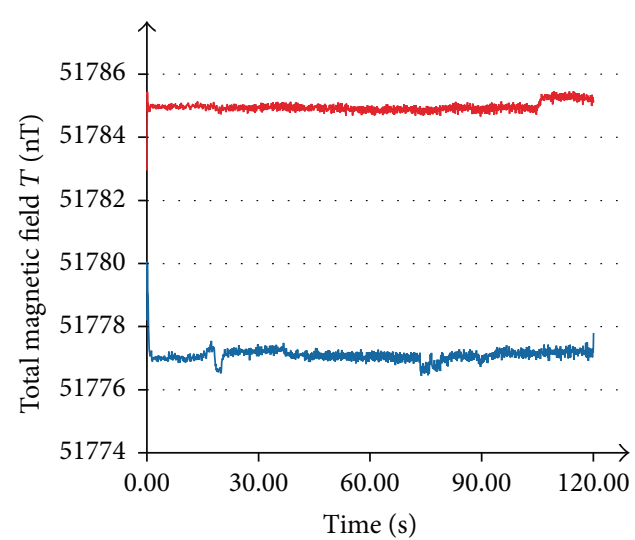

(b)

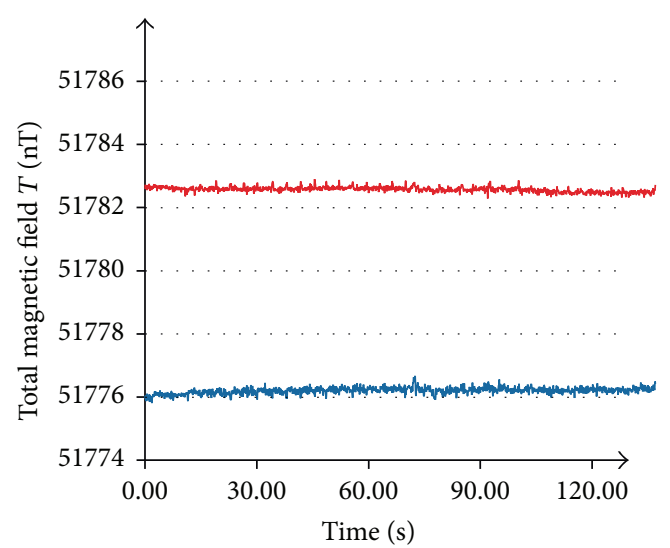

(d)

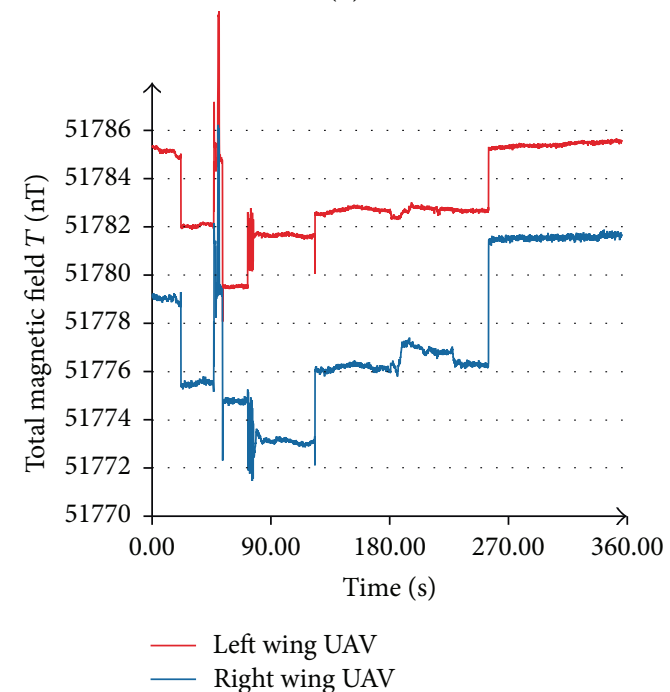

(f)

\begin{tabular}{lccccccc}
\hline & Mode & $\begin{array}{c}\text { Medium } \\
\text { engine power (a) }\end{array}$ & $\begin{array}{c}\text { Maximum } \\
\text { engine power (b) }\end{array}$ & $\begin{array}{c}\text { Medium engine power } \\
\text { and servos (c) }\end{array}$ & Only servos (d) & $\begin{array}{c}\text { Varied } \\
\text { engine power (e) }\end{array}$ & $\begin{array}{c}\text { Power } \\
\text { off (f) }\end{array}$ \\
\hline \multirow{2}{*}{ Left wing } & Min (nT) & 51784.2 & 51782.9 & 51783.2 & 51782.3 & 51780.34 & 51778.1 \\
UAV & Max (nT) & 51786.3 & 51785.5 & 51789.2 & 51782.9 & 51787.57 & 51790.9 \\
& Mean (nT) & 51785.1 & 51785.0 & 51785.0 & 51782.6 & 51785.26 & 51783.3 \\
\hline \multirow{2}{*}{ Right wing } & Min (nT) & 51778.4 & 51776.4 & 51777.1 & 51775.8 & 51774.35 & 51771.5 \\
UAV & Max (nT) & 51780.4 & 51780.1 & 51784.4 & 51776.7 & 51782.27 & 51786.2 \\
& Mean (nT) & 51779.5 & 51777.1 & 51779.3 & 51776.2 & 51779.21 & 51777.5 \\
\hline
\end{tabular}

FIGURE 5: Magnetic field at the ends of UAV's wings at different modes: (a) medium engine power; (b) maximum engine power; (c) medium engine power and servos; (d) only sensors; (e) varied engine power; (f) power off. 


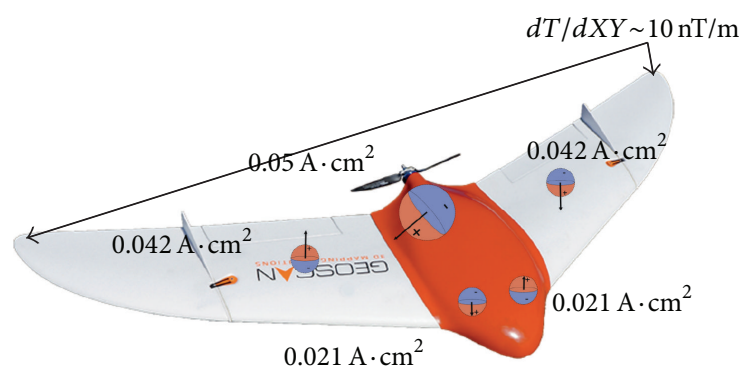

FIGURE 6: Location of UAV magnetic noise sources.

where $\bar{M}$ is dipole and $\bar{R}$ is vector directed from the center of the source to the measuring point. The magnetic field of a sphere at point $(x, y)$ is [8]

$$
\begin{aligned}
X_{a} & =\left(\frac{M}{r^{5}}\right)\left[\left(2 x^{2}-y^{2}-h^{2}\right) \cos i \cos A\right. \\
& +3 x(y \cos i \sin A-h \sin i)], \\
Y_{a} & =\left(\frac{M}{r^{5}}\right)\left[\left(2 y^{2}-x^{2}-h^{2}\right) \cos i \sin A\right. \\
& +3 y(y \cos i \cos A-h \sin i)], \\
Z_{a} & =\left(\frac{M}{r^{5}}\right)\left[\left(2 h^{2}-x^{2}-y^{2}\right) \cos i\right. \\
& -3 h \cos i(x \cos A-y \sin A)],
\end{aligned}
$$

where $V$ is a volume, $H$ is a depth of the center, $J$ is a magnetization vector, $M=J V$ is magnetic moment, $i$ is an inclination of the magnetization vector, $A$ is the angle between the projections $J$ on the $x$-axis and the $x 0 y$-plane, and $r=\sqrt{x^{2}+y^{2}+h^{2}}$. Using (2), the anomalous magnetic field $\Delta T$ is equal to [8]

$$
\Delta T=Z_{a} \sin I+H_{a} \cos I \cos A_{0},
$$

where $I$ is magnetic inclination, $A_{0}$ is magnetic azimuth of vector $H_{a}$, and $H_{a}=\sqrt{X_{a}^{2}+Y_{a}^{2}}$.

The electroengine can be represented by a dipole with magnetic moment $M=0.05 \mathrm{~A} \cdot \mathrm{cm}^{2}$, for servos $M=$ $0.042 \mathrm{~A} \cdot \mathrm{cm}^{2}$ and for frontal elements $M=0.021 \mathrm{~A} \cdot \mathrm{cm}^{2}$. For each dipole, magnetic field at a map was calculated using formula (3) and superposition of them is assumed as the final magnetic map. The resulting magnetic map represents good approximation with experimental data (Figure 7(a)). The gradient of calculated magnetic field at the ends of wings is about $10 \mathrm{nT} / \mathrm{m}$, which is also comparable with the observed values. As noted above, the dynamic experiments have shown unfitness of the UAV with electroengine for magnetic survey. Replacement of an electric engine with combustion one in the mathematical model (Figure 7(b)) demonstrates horizontal gradient of the magnetic field at the end of a wing below $1 \mathrm{nT} / \mathrm{m}$. However, the combustion engine fitting the task has weight $700 \mathrm{~g}$, versus $284 \mathrm{~g}$ of electroengine. This, along with the additional weight of a sensor (up to $200 \mathrm{~g}$ ) at the end

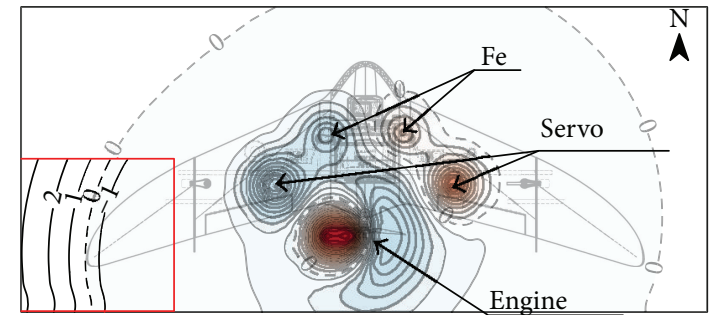

(a)

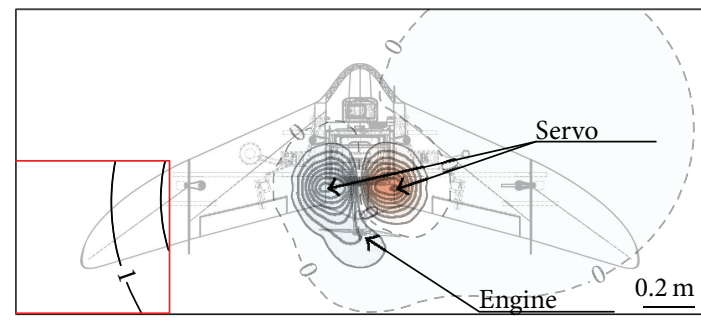

Contours step: $50 \mathrm{nT}$

(in red box: $1 \mathrm{nT}$ )

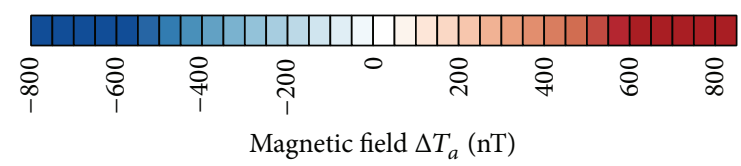

(b)

FIGURE 7: Modeled magnetic field: (a) UAV; (b) NUAP.

of a wing, makes UAV "Geoscan-201" unstable because of displacing center of mass.

The aerodynamic mathematical model of NUAP, equipped with combustion engine and sensor, was calculated using XFLR5 software [9]. The model's weight is $8 \mathrm{~kg}$, and cruise speed is about $20 \mathrm{~m} / \mathrm{s}$. The results of modeling demonstrate the best position of the center mass point located at $900-925 \mathrm{~mm}$ from front edge, and optimal angle of attack is 5 degrees.

\section{Conclusions}

The recommendations following from aerodynamic and magnetic models are implemented in the design of NUAP (Figure 8). The NUAP flight duration is at least 5 hours at the altitude of $20-50 \mathrm{~m}$ with $20 \mathrm{~m} / \mathrm{s}$ operation speed. The concept of NUAP equipped with one or two atomic magnetic sensors is expected to have an error of the magnetic survey below $2 \mathrm{nT}$, which completely facilitates magnetic survey of $1: 5,000-1: 50,000$ scale. The advantage of this concept is

(1) no special prefield works (flights with different azimuth, typically 8 , for magnetic deviation studies),

(2) no additional fluxgate magnetometer,

(3) no special software for postprocessing and deviation compensation,

(4) autonomy of flight (5 hours versus 1,5-2 for UAV with electric engine). 


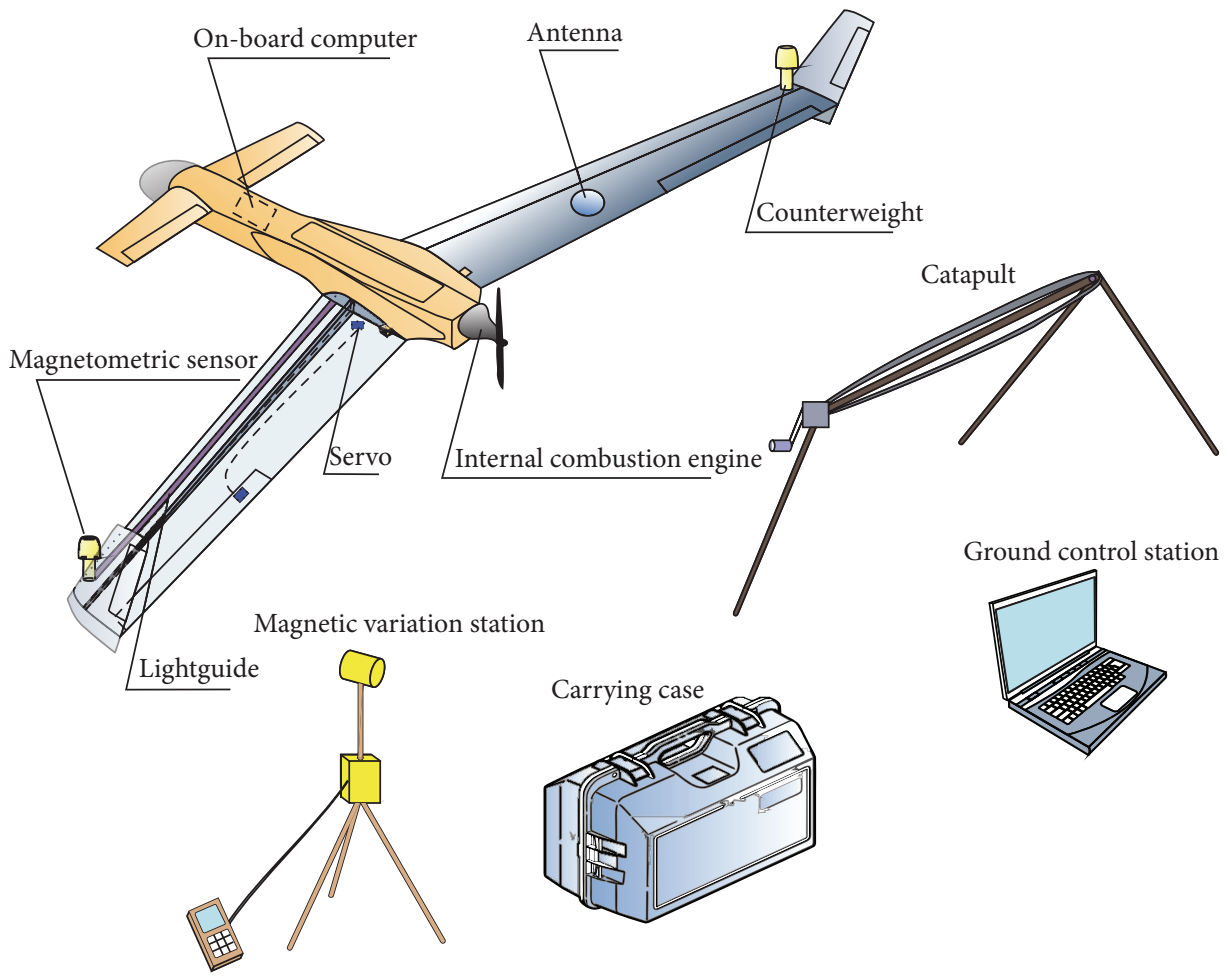

FIgURE 8: The concept of the NUAP.

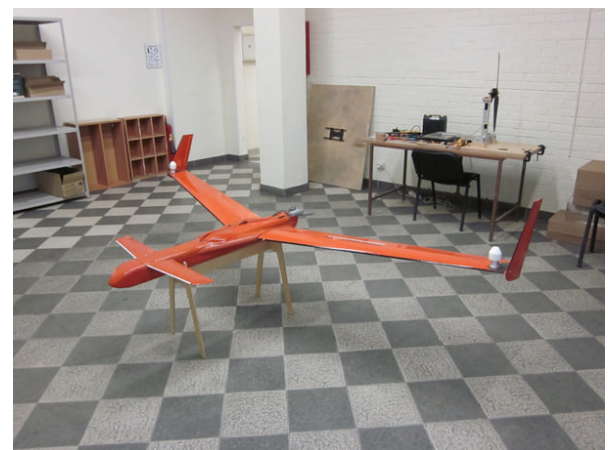

FIGURE 9: The NUAP prototype.

Thus, the use of NUAP based on the ultralight UAV can sufficiently facilitate aerial magnetic survey, which is especially important for the areas below 200 sq. $\mathrm{km}$. In addition to the magnetometer, the NUAP can be equipped with multispectral or hyperspectral camera, which opens even wider opportunities for resolving geological and engineering tasks. Using the developed concept, the Geoscan group has created NUAP prototype (Figure 9).

\section{Competing Interests}

The authors declare that there are no competing interests regarding the publication of this paper.

\section{Acknowledgments}

This research was funded by the Ministry of Education and Science of Russian Federation, under Agreement no. 14.607.21.0081 (ID no. RFMEFI60714X0081).

\section{References}

[1] R. Versteeg, M. McKay, M. Anderson, R. Johnson, B. Selfridge, and J. Bennett, "Feasibility study for an autonomous UAVmagnetometer system," Final Rep. SERDP SEED 1509:2206, Idaho National Lab, Idaho Falls, Idaho, USA, 2007.

[2] R. Forrester, M. S. Huq, M. Ahmadi, and P. Straznicky, "Magnetic signature attenuation of an unmanned aircraft system for aeromagnetic survey," IEEE/ASME Transactions on Mechatronics, vol. 19, no. 4, pp. 1436-1446, 2014.

[3] C. Samson, P. Straznicky, J. Laliberte, R. Caron, S. Ferguson, and R. Archer, "Designing and building an unmanned aircraft system for aeromagnetic surveying," in Proceedings of the SEG 80th Annual International Meeting, SEG Expanded Abstracts, pp. 1167-1171, Denver, Colo, USA, 2010.

[4] R. M. Caron, C. Samson, P. Straznicky, S. Ferguson, and L. Sander, "Aeromagnetic surveying using a simulated unmanned aircraft system," Geophysical Prospecting, vol. 62, no. 2, pp. 352363, 2014.

[5] J. B. Stoll, "Unmanned aircraft systems for rapid near surface geophysical measurements," International Archives of the Photogrammetry, Remote Sensing and Spatial Information SciencesF, vol. XL-1/W2, UAV-g2013, pp. 391-394, 2013.

[6] L. E. Zaffanella, T. P. Sullivan, and I. Visintainer, "Magnetic field characterization of electrical appliances as point sources 
through in situ measurements," IEEE Transactions on Power Delivery, vol. 12, no. 1, pp. 443-449, 1997.

[7] J. R. Reitz and F. J. Milford, Foundation of Electromagnetic Theory, Addison-Wesley, 1967.

[8] A. Logachev and V. P. AZakharov, Magnetic Prospecting, Nedra, Leningrad, Russia, 1979 (Russian).

[9] A. Deperrois, Guidelines for XFLR5 v6.03, 2011, http:// sourceforge.net/projects/xflr5/files/xflr5\%20v6.03\%20Beta/. 

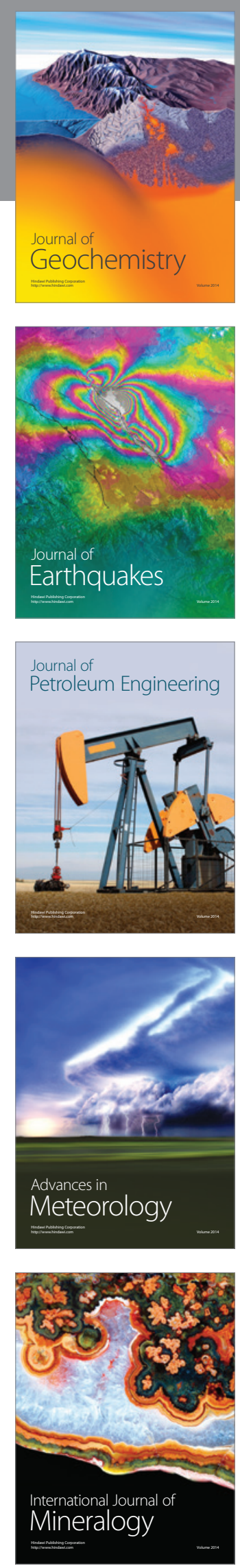
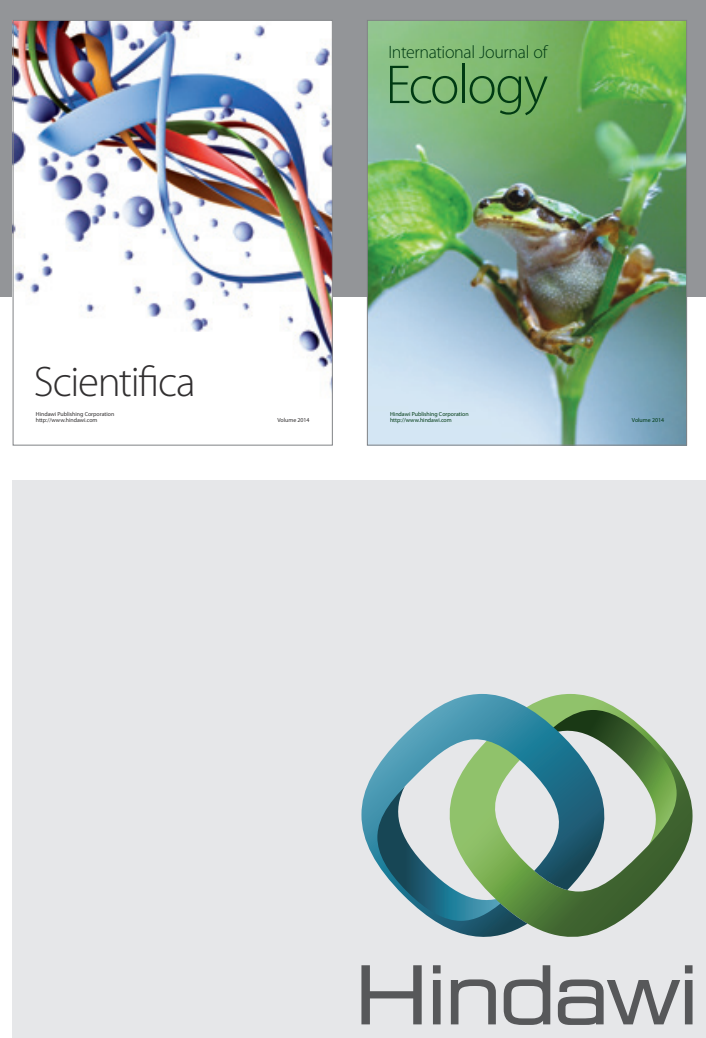

Submit your manuscripts at

http://www.hindawi.com
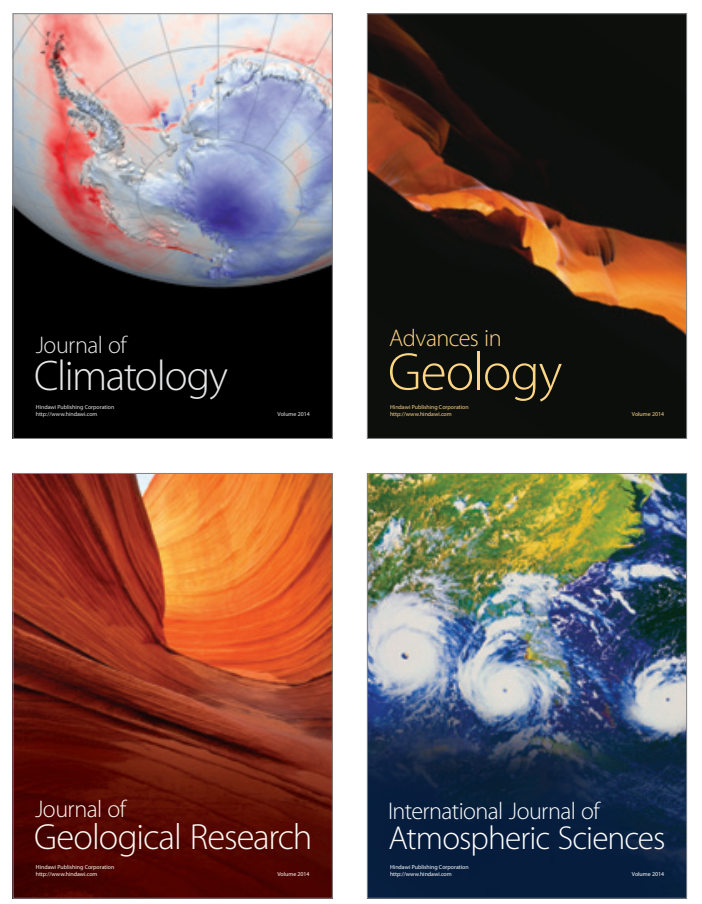

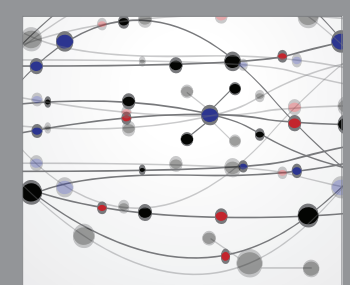

The Scientific

\section{World Journal}
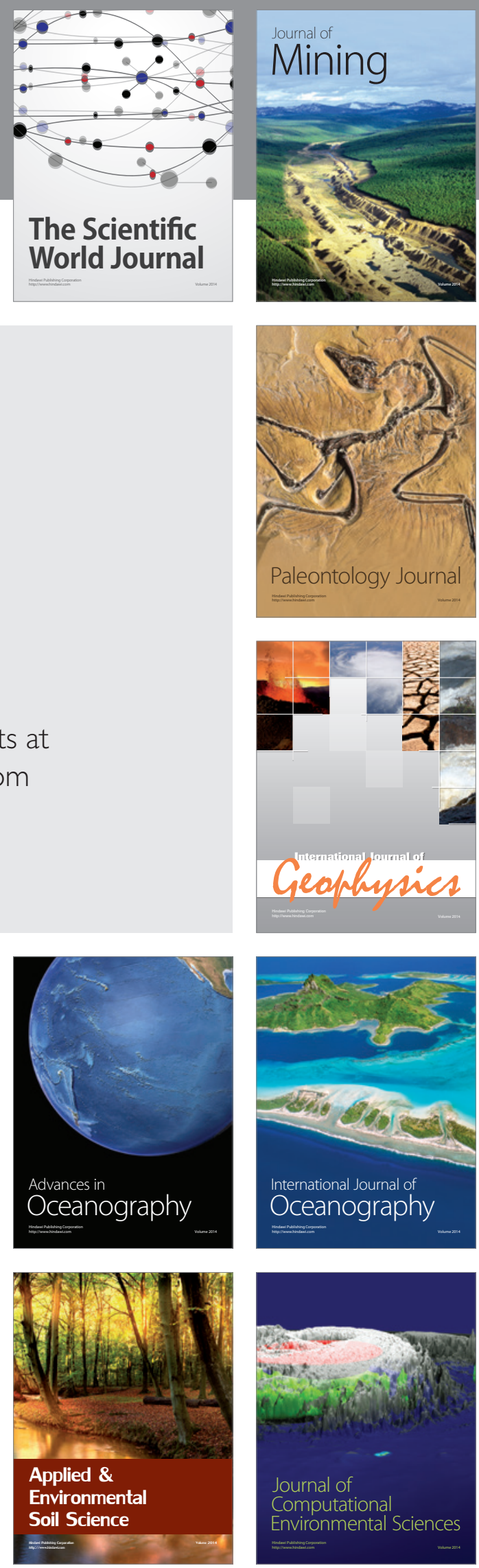